\title{
THE YOUNG MEASURE METHOD FOR A NONLINEAR VARIATIONAL WAVE EQUATION
}

\author{
Ping ZhaNG ${ }^{\dagger}$ YUXI ZheNG ${ }^{\ddagger}$
}

\begin{abstract}
A nonlinear wave equation arises in a simplified liquid crystal model through the variational principle. The wave speed of the wave equation is a given function of the wave amplitude. Hunter and Saxton has derived a simple asymptotic equation for weakly nonlinear unidirectional waves of the nonlinear wave equation. Previous work has established the existence of weak solutions to the initial value problem for the asymptotic equation for data in the space of bounded variations. We improve the previous work to the natural space of square integrable functions, and we establish the uniqueness of weak solutions for both the dissipative and conservative types. Our main point here is to show the new method called Young measure method which does not need bounded derivatives for compactness.

We also have results on the full nonlinear wave equation. It has been known from joint work with Glassey and Hunter for the equation that smooth initial data may develop singularities in finite time, a sequence of weak solutions may develop concentrations, while oscillations may persist. We formulate a viscous approximation of the equation and establish the global existence of smooth solutions for the viscously perturbed equation. For monotone wave speed functions in the equation, we find an invariant region in the phase space in which we discover: (a) smooth data evolve smoothly forever; (b) both the viscous regularization and the smooth solutions obtained through data mollification and step (a) for not-as-smooth initial data yield weak solutions to the Cauchy problem of the nonlinear variational wave equation. The main tool is the Young measure theory and related techniques.
\end{abstract}

More specifically, we study the Cauchy problem for the nonlinear wave equation

$$
\left\{\begin{array}{l}
u_{t t}-c(u)\left[c(u) u_{x}\right]_{x}=0, \quad t>0, x \in \mathbb{R} \\
\left.\left(u, \partial_{t} u\right)\right|_{t=0}=\left(u_{0}, u_{1}\right)(x) \in\left(H^{1}, L^{2}\right)
\end{array}\right.
$$

The latest results are

(1) On the asymptotic equation, we have existence and uniqueness of multiple weak solutions in the weak norm $L^{2}$ of the derivative $u_{x}$.

(2) For the nonlinear wave equation, we found some invariant regions and some global smooth solutions.

1. Origin of the variational wave equation. For nematic liquid crystals $([\mathrm{CGH}][\mathrm{EK}][\mathrm{V}])$, there is this Oseen-Frank potential energy

$$
W(\mathbf{n}, \nabla \mathbf{n})=\alpha|\mathbf{n} \times(\nabla \times \mathbf{n})|^{2}+\beta(\nabla \cdot \mathbf{n})^{2}+\gamma(\mathbf{n} \cdot \nabla \times \mathbf{n})^{2},
$$

where $\mathbf{n} \in \mathbb{S}^{2}$ denotes the mean orientation field of the long molecules of the crystal, while the positive constants $\alpha, \beta$, and $\gamma$ are elastic constants of the liquid crystal. For the special case $\alpha=\beta=\gamma$, the potential energy density reduces to

$$
W(\mathbf{n}, \nabla \mathbf{n})=\alpha|\nabla \mathbf{n}|^{2},
$$

which is the potential energy density used in harmonic maps into the sphere $\mathbb{S}^{2}$. Similar to studies in harmonic maps, there are three types of equations associated with (2):

\footnotetext{
$\dagger^{\dagger}$ Academia Sinica, Beijing, 100080, China.

$\ddagger$ Department of Mathematics, The Pennsylvania State University, University Park, PA 16802, USA.
} 
the elliptic, parabolic, and hyperbolic equations:

$$
\begin{aligned}
& -\frac{\delta \mathcal{W}}{\delta \mathbf{n}}+\lambda \mathbf{n}=0 \\
& \mathbf{n}_{t}=-\frac{\delta \mathcal{W}}{\delta \mathbf{n}}+\lambda \mathbf{n} \\
& \mathbf{n}_{t t}=-\frac{\delta \mathcal{W}}{\delta \mathbf{n}}+\lambda \mathbf{n} .
\end{aligned}
$$

Equation (1) is the hyperbolic type in one space dimension. See [GH2] for more background information.

For the one-dimensional case where $\mathbf{n}$ is restricted to $\mathbb{S}^{1}$ and $u$ is used to represent the angle of $\mathbf{n}$ with the $x$-axis (see Figure 1), the potential reduces to

$$
\mathcal{W}=c^{2}(u)\left(\partial_{x} u\right)^{2}
$$

where

$$
c^{2}(u)=\alpha \cos ^{2} u+\beta \sin ^{2}(u) .
$$

The equation in (1) is the Euler-Lagrange equation of the least action principle

$$
\frac{\delta}{\delta u} \iint\left\{\left(\partial_{t} u\right)^{2}-c^{2}(u)\left(\partial_{x} u\right)^{2}\right\} d x d t=0
$$

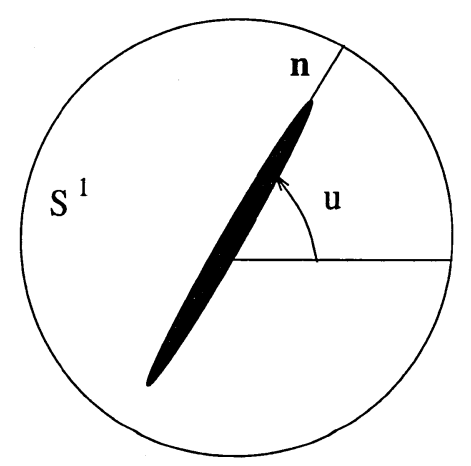

Figure 1. The angle of the molecule.

2. Asymptotic equations. Despite its simplicity, equation (1) is not easy. A study of its geometric optical solutions is helpful and interesting. Look for solutions of the form:

$$
\psi(t, x)=u_{0}+\epsilon u\left(\epsilon t, x-c_{0} t\right)+O\left(\epsilon^{2}\right)
$$

where $u_{0}$ is a constant state and $c_{0}=c\left(u_{0}\right)>0$ is its speed, Hunter and Saxton ([HS]) found that $u(\cdot, \cdot)$ satisfies

$$
\left(u_{t}+u u_{x}\right)_{x}=\frac{1}{2} u_{x}^{2}
$$


up to a scaling factor, assuming that $c^{\prime}\left(u_{0}\right) \neq 0$.

If $u_{0}$ is such that $c^{\prime}\left(u_{0}\right)=0, c^{\prime \prime}\left(u_{0}\right) \neq 0$, then the equation is

$$
\left(u_{t}+u^{2} u_{x}\right)_{x}=u u_{x}^{2} \text {. }
$$

In general if $u_{0}$ is such that $c^{(k)}\left(u_{0}\right)=0, k=1,2 \ldots n-1$, but $c^{(n)}\left(u_{0}\right) \neq 0$, the equation is

$$
\left(u_{t}+u^{n} u_{x}\right)_{x}=\frac{1}{2} n u^{n-1} u_{x}^{2} .
$$

Another form of the first asymptotic equation is

$$
\partial_{t} v+\partial_{x}(u v)=\frac{1}{2} v^{2}, \quad u_{x}=v
$$

We shall not study the second and higher asymptotic equations in this short talk.

3. Phenomena for asymptotic equations. From joint work with Hunter ([HZ]), we know that the first asymptotic equation has singularity formation (mild blow-up) and the solution can be continued beyond singularity formation by two different ways resulting in the dissipative and conservative solutions, see Figure 2, where the solution is continued either by zero (not shown in the figure) or the decaying solution beyond $t^{*}$. We explain that the data in Figure 2 is that $v(0, x)=-1$ for $x \in[0,1]$ and zero otherwise. The blow-up at $t=t^{*}$ is such that $v \rightarrow-\infty$. The support of $v(t, x)$ shrinks to a point at the precise rate that the $L^{2}$ norm of $v(t, \cdot)$ is constant.

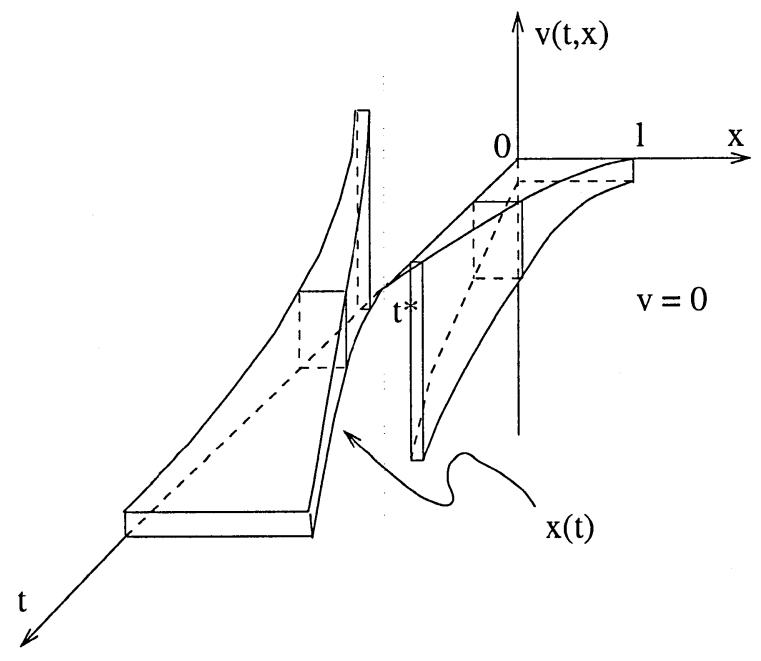

Figure 2. A conservative solution.

The first asymptotic equation also has persistence of oscillation, which we choose not to discuss here. 
4. Classical existence of solutions. We can establish the a priori estimate: $L^{2}$ norm of $v$ is non-increasing in time.

If the initial $v(x, 0)$ has bounded total variation, then the global existence of weak solutions has been established in joint work with Hunter [HZ], by removing small sets of singularity in the approximate solutions.

5. Compensated compactness. It is natural to ask for the existence result in the space $L^{2}$, in view of the a priori estimate. Let us rewrite (3) and consider the initial-boundary value problem

$$
\begin{cases}v_{t}+(u v)_{x}=\frac{1}{2} v^{2}, & u_{x}=v, \quad(x>0, t>0) \\ v(x, 0)=v_{0}(x), & u(0, t)=0 .\end{cases}
$$

Suppose there is a sequence of approximate solutions $\left\{v^{n}, u^{n}\right\}$ so that

$$
v_{t}^{n}+\left(u^{n} v^{n}\right)_{x}=\frac{1}{2}\left(v^{n}\right)^{2}, \quad u_{x}^{n}=v^{n}
$$

We would like to use compensated compactness to derive strong compactness for $v^{n}$ in $L^{2}$. So we multiply (5) with $v^{n}$ to obtain

$$
\left[\left(v^{n}\right)^{2}\right]_{t}+\left[u^{n}\left(v^{n}\right)^{2}\right]_{x}=0 .
$$

Suppose the right-hand side of (5) is compact in $\mathrm{H}^{-1}$. However the determinant of the four terms on the left-hand sides of (5) and (6) is always zero. So compensated compactness fails here. For the frame work of compensated compactness, see Tartar [T].

6. Young measure method (formal proof of existence in $L^{2}$ ). We let

$$
\left(u^{n}, v^{n},\left(v^{n}\right)^{2}\right) \rightarrow(\bar{u}, \bar{v}, \bar{w}) .
$$

We know immediately that

$$
\bar{w} \geq(\bar{v})^{2}
$$

from functional analysis. On the other hand, we can find a closed system of equations for the three quantities $\{\bar{u}, \bar{v}, \bar{w}\}$ :

$$
\begin{aligned}
\partial_{t} \bar{v}+\partial_{x}(\bar{u} \bar{v}) & =\frac{1}{2} \bar{w} \\
\partial_{t} \bar{w}+\partial_{x}(\bar{u} \bar{w}) & =0 \\
\partial_{x} \bar{u} & =\bar{v} .
\end{aligned}
$$

To compare the two quantities $\bar{w}$ and $\bar{v}^{2}$ using the equations, we multiply the $\bar{w}$ equation with $\frac{1}{2} \bar{w}^{-1 / 2}$ to derive

$$
\partial_{t} \bar{w}^{1 / 2}+\partial_{x}\left(\bar{u} \bar{w}^{1 / 2}\right)=\frac{1}{2} \bar{v} \bar{w}^{1 / 2}
$$

whose right-hand side is less than that in the equation for $\bar{v}$, in view of $\bar{w} \geq(\bar{v})^{2}$. With same initial data for $(\bar{w})^{\frac{1}{2}}$ and $\bar{v}$ due to strong convergence of the initial approximations, it follows from the equations that the reverse inequality

$$
\bar{w} \leq \bar{v}^{2}
$$


holds. So

$$
\bar{w}=\bar{v}^{2}
$$

which implies strong convergence of the sequence $\left\{v^{n}\right\}$ in $L^{2}$.

For the rigorous proof, see [ZZ1]. For Young measures, see Gérard [G]. For similar applications of Young measures, see Lions [L2].

\section{Uniqueness of solutions.}

(A) For nonnegative data, the dissipative and conservative solutions are the same. And they are unique. The method is mollification, and the uniqueness of the inverse flow of characteristics is essential. See [ZZ2].

(B) For general $L^{2}$ data, the inverse flow of characteristics is not unique. We follow characteristics and estimate sets of singularities. The criteria are: Conservative solutions conserve local energy between any pair of characteristics; Dissipative solutions have upper bound $v \leq \frac{2}{t}$. Details are in [ZZ3].

We comment on mollification for uniqueness. Mollify the equation

$$
\partial_{t} v+u \partial_{x} v=-\frac{1}{2} v^{2}, \quad u_{x}=v
$$

by $* \rho^{\epsilon}$ to yield

$$
\partial_{t} v^{\epsilon}+u \partial_{x} v^{\epsilon}=-\frac{1}{2}\left(v^{2}\right)^{\epsilon}+R^{\epsilon}, \quad u_{x}=v
$$

where $R^{\epsilon}=u \partial_{x} v^{\epsilon}-\left(u \partial_{x} v\right)^{\epsilon}$. Then follow characteristics, find $v^{\epsilon}$ and pass limit $\epsilon \rightarrow 0$. DiPerna-Lions folklore Lemma on commutator ([DL] or [L1])

$$
u \cdot \nabla v^{\epsilon}-(u \cdot \nabla v)^{\epsilon} \rightarrow 0
$$

is handy.

8. Blow-up of smooth solutions of the wave equation. This is from joint work with Glassey and Hunter [GHZ].

The idea is to choose data close to the regime covered by the asymptotic equation (i.e., small amplitude with high frequency). Show blow-up before $c^{\prime}$ changes sign.

Sketch of blow-up of the wave equation:

Assume no blow-up.

Take any $u_{0}$ such that $c^{\prime}\left(u_{0}\right) \neq 0$.

Choose peaked data near $u_{0}$ with large $S=O(1 / \epsilon)$ and small $R$. See Figure 3 . Here $R$ and $S$ are the "Riemann invariants" (see Section 10 later). And $\epsilon$ is the small amplitude.

Use energy in a cone to control the integral of $R^{2}$.

Then estimate growth of $u$ from $u_{0}$ (which is $O(\sqrt{t \epsilon})$ ) to control the sign of $c^{\prime}$.

Then $S$ satisfies a Ricatti equation, which blows up at $O(1)$ time.

9. Oscillation/annihilation phenomena of the wave equation. The wave equation has weak formulation

$$
u_{t t}-\left(c^{2} u_{x}\right)_{x}=-c c^{\prime}\left(u_{x}\right)^{2} .
$$

It seems need strong compactness of $u_{x}$ in $L^{2}$. But example shows 


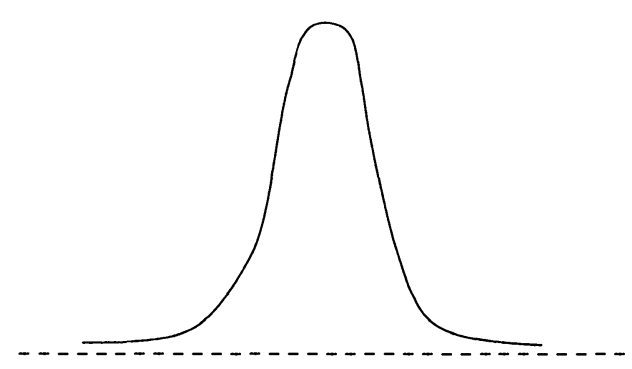

Figure 3. Initial Data for blow-up.

$$
\begin{array}{rlrl}
u^{n} & \rightarrow \frac{\pi}{2} \quad \text { uniformly, } \\
u_{x}^{n} \rightarrow 0 \quad \text { weakly in } L^{2}, \\
\left(u_{x}^{n}\right)^{2} \rightarrow \alpha \neq 0 \quad \text { weakly in } L^{1}, \\
c^{\prime}\left(u^{n}\right)\left(u_{x}^{n}\right)^{2} \rightarrow 0 \quad \text { weakly in } L^{1} .
\end{array}
$$

So the weak limit (the constant $\frac{\pi}{2}$ ) is still a weak solution despite lack of convergence.

10. An invariant region of the wave equation. We always assume

$$
0<C_{1} \leq c(\cdot) \leq C_{2} \text {, and }\left|c^{(l)}(\cdot)\right| \leq M_{l}
$$

for $l \geq 1$. We also assume in most cases that

$$
c^{\prime}(\cdot) \geq 0
$$

In some cases we even assume

$$
c^{\prime}(u) \geq C_{M}>0, \quad u \in[-M, M] .
$$

Let

$$
\begin{aligned}
& R:=\partial_{t} u+c(u) \partial_{x} u, \quad S:=\partial_{t} u-c(u) \partial_{x} u, \\
& \tilde{c}(\cdot):=\frac{1}{4} \ln c(\cdot),
\end{aligned}
$$

so that $\tilde{c}^{\prime}(u)=c^{\prime}(u) /[4 c(u)]$. Then the wave equation can be written in the $\mathrm{n} \epsilon$

$$
\left\{\begin{array}{l}
\partial_{t} R-c \partial_{x} R=\tilde{c}^{\prime}(u)\left(R^{2}-S^{2}\right), \\
\partial_{t} S+c \partial_{x} S=\tilde{c}^{\prime}(u)\left(S^{2}-R^{2}\right), \\
\partial_{x} u=\frac{R-S}{2 c(u)} .
\end{array}\right.
$$

Lemma (Existence of invariant Region). Assume $c^{\prime} \geq 0, R(0, a$ $S(0, x) \leq 0$. Then $R(t, x) \leq 0$ and $S(t, x) \leq 0$. 
For a proof, one needs to consider

$$
\left\{\begin{array}{l}
\partial_{t} R_{\epsilon}-c\left(u_{\epsilon}\right) \partial_{x} R_{\epsilon}=\tilde{c}^{\prime}\left(u_{\epsilon}\right)\left(R_{\epsilon}^{2}-S_{\epsilon}^{2}\right)+\epsilon \partial_{x}^{2} R_{\epsilon} \\
\partial_{t} S_{\epsilon}+c\left(u_{\epsilon}\right) \partial_{x} S_{\epsilon}=\tilde{c}^{\prime}\left(u_{\epsilon}\right)\left(S_{\epsilon}^{2}-R_{\epsilon}^{2}\right)+\epsilon \partial_{x}^{2} S_{\epsilon} \\
\partial_{x} u_{\epsilon}=\frac{R_{\epsilon}-S_{\epsilon}}{2 c\left(u_{\epsilon}\right)}
\end{array}\right.
$$

see [ZZ4]. There exists a global smooth solution for the Cauchy problem. There holds the maximum principle.

This result contrasts sharply with the blow-up phenomena of Fujita $[\mathrm{F}]$ for

$$
\partial_{t} V=\frac{1}{2} V^{2}+\epsilon \partial_{x}^{2} V
$$

with any initial data $V_{0}(x)$ such that $V_{0}\left(x_{0}\right)>0$ for some point $x_{0}$.

11. Rarefactive solutions of the wave equation.

Denote $R_{0}=R(0, x), S_{0}=S(0, x)$.

Theorem 1 (Global rarefactive $L^{p}$ solutions). Assume $c^{\prime} \geq 0, R_{0} \leq$ $0, S_{0} \leq 0$, and $\left(R_{0}, S_{0}\right) \in L^{p}(\mathbb{R})$ with compact support for some $p>3$. Then (1) has a global admissible weak solution. The solution can be obtained either through initial data mollification or vanishing viscosity. Moreover, there holds $(R, S)(t, x) \in$ $L^{\infty}\left(\mathbb{R}^{+}, L^{p}(\mathbb{R})\right)$. Furthermore, if $(7)$ holds, then $\partial_{x} u(t, x) \in L^{p+1}([0, T] \times \mathbb{R})$ for any $T>0$.

Theorem 2 (Global rarefactive $L^{2}$ solutions). Assume (7) holds and $R_{0} \leq 0, S_{0} \leq 0,\left(R_{0}, S_{0}\right) \in L^{2}(\mathbb{R})$ with compact support. Then (1) has a global admissible weak solution. Moreover, there holds $\partial_{x} u \in L^{2+\alpha}([0, T] \times \mathbb{R})$ for any $\alpha<1$ and $T<\infty$. Furthermore there exists a constant $C>0$ such that

$$
-\frac{C}{t} \leq R(t, x) \leq 0, \quad-\frac{C}{t} \leq S(t, x) \leq 0,
$$

$t \in(0,1], x \in \mathbb{R}$, and $(R, S)$ remain bounded from below by $-C$ for all time $t>1$.

For proofs of Theorems 1-2, see [ZZ4].

This talk was also given at the AMS regional meeting, Gainesville, Florida, the International Conference on Partial Differential Equations at the University of Iowa, Iowa City, and the Hyperbolic Aspect of Fluids at Oberwolfach, Spring, 1999.

Acknowledgements: The work of Ping Zhang is supported by the Chinese Post-Doctor's Foundation and Morningside Center of Mathematics, and that of Yuxi Zheng is supported in part by NSF DMS-9703711 and the Alfred P. Sloan Research Fellows award.

\section{REFERENCES}

[CGH] J. Coron, J. Ghidaglia, And F. HÉlein (EDs.), Nematics, Kluwer Academic Publishers, 1991.

[DL] R. J. DiPERNA AND P. L. LIONS, Ordinary differential equations, transport theory and Sobolev spaces, Invent. Math., 98 (1989), pp. 511-547. 
[EK] J. L. ERICKSEN AND D. Kinderlehrer (EDS.), Theory and Application of Liquid Crystals, IMA Volumes in Mathematics and its Applications, Vol. 5 Springer-Verlag, New York (1987).

[F] H. FUJITA, On the blowing up of solutions of the Cauchy problem for $u_{t}=\Delta u+u^{1+\alpha}, \mathrm{J}$. Fac. Sci. Tokyo Sect. IA, Math., 13 (1966), pp. 109-124.

[G] P. GÉrard, Microlocal Defect Measures, Comm. Partial Differential Equations, 16 (1991), pp. 1761-1794.

[GHZ] R. T. Glassey, J. K. Hunter AND YUXI Zheng, Singularities of a variational wave equation, J. Diff. Eq., 129 (1996), pp. 49-78.

[GH2] R. T. Glassey, J. K. Hunter AND YUXI Zheng, Singularities and oscillations in a nonlinear variational wave equation, Singularities and Oscillations, edited by J. Rauch and M. E. Taylor, IMA, Vol. 91, Springer, 1997.

[HS] J. K. Hunter and R. A. Saxton, Dynamics of director fields, SIAM J. Appl. Math., 51 (1991), pp. 1498-1521.

[HZ] J. K. HUNTER AND YUXI Zheng, On a nonlinear hyperbolic variational equation I and II, Arch. Rat. Mech. Anal., 129 (1995), pp. 305-353 and 355-383.

[L1] P. L. Lions, Mathematical topics in fluid mechanics, Vol. 1, Incompressible Models, Lecture series in mathematics and its applications, v.3, Clarendon Press, Oxford, 1996.

[L2] P. L. Lions, Mathematical topics in fluid mechanics, Vol. 2, Compressible Models, Lecture series in mathematics and its applications, v.6, Clarendon Press, Oxford, 1998.

[T] L. TARTAR, Compensated compactness and applications to partial differential equations, Nonlinear Analysis and Mechanics: Heriot-Watt Symposium (R. J. Knops, ed.), Research Notes in Math., Vol. 39, Pitman Press, 1979.

[V] E. Virga, Variational Theories for Liquid Crystals, Chapman \& Hall, New York (1994).

[ZZ1] PING Zhang AND YUXi Zheng, On oscillations of an asymptotic equation of a nonlinear variational wave equation, Asymptotic Analysis, 18 (1998), pp. 307-327.

[ZZ2] PING ZHANG AND YUXI ZHENG, On the existence and uniqueness of solutions to an asymptotic equation of a variational wave equation, Acta Mathematica Sinica (English Series), 15 (1999), pp. 115-130.

[ZZ3] PING ZhANG AND YUXI Zheng, Existence and uniqueness of solutions to an asymptotic equation of a variational wave equation with general initial data, Arch. Rat. Mech. Anal., 155 (2000), pp. 49-83.

[ZZ4] PING Zhang AND YUXI Zheng, Rarefactive solutions to a nonlinear variational wave equation of liquid crystals, Commun. Partial Differential Equations, 26 (2001), pp. 381-419.

Added in galley proof in May 2002: These are articles we have written since the talk was given.

1. On the Second-Order Asymptotic Equation of a Variational Wave Equation, Proceedings of the Royal Society of Edinburgh, A. Mathematics, (in press), 2001.

2. Singular and Rarefactive Solutions to a Nonlinear Variational Wave Equation of Liquid Crystals, Chinese Annals of Math., 22B (2001), pp. 159-170.

3. Weak Solutions to a Nonlinear Variational Wave Equation of Liquid Crystals, to be submitted for publication, preprint, 2000. 